\title{
Pharmacokinetic Properties of Rivaroxaban in Healthy Human Subjects
}

\author{
Sosipatros Bratsos ${ }^{1}$ \\ 1. Cardiology, Imperial College London, London, GBR
}

Corresponding author: Sosipatros Bratsos, sb3113@ic.ac.uk

\begin{abstract}
Direct oral anticoagulants (DOACs) have predictable pharmacokinetics and pharmacodynamics, limited potential for drug to drug interactions, and can be given at fixed doses without the need for routine coagulation monitoring, which makes them a very attractive alternative to vitamin $\mathrm{K}$ antagonists. DOACs act by specifically targeting a single coagulation factor, such as Factor Xa or thrombin. Rivaroxaban is a direct Factor Xa inhibitor and has been approved for use in several thromboembolic disorders, such as the prevention of stroke and systemic embolism in adults with non-valvular atrial fibrillation and the prevention of recurrent deep vein thrombosis and pulmonary embolism in adult patients. This review aimed to provide an overview of the mechanism of action of rivaroxaban and outline its pharmacokinetic properties (absorption, distribution, metabolism, and excretion) in healthy adult subjects.
\end{abstract}

Received 08/15/2019

Review began 08/16/2019

Review ended 08/17/2019

Published 08/25/2019

๑) Copyright 2019

Bratsos. This is an open access article distributed under the terms of the Creative Commons Attribution License CC-BY 3.0., which permits unrestricted use, distribution, and reproduction in any medium, provided the original author and source are credited.
Categories: Cardiology, Miscellaneous, Other

Keywords: rivaroxaban, pharmacokinetics

\section{Introduction And Background}

Direct oral anticoagulants (DOACs) have recently been developed to overcome the limitations of established oral anticoagulants such as vitamin K antagonists (VKAs). In contrast to VKAs, DOACs tend to have predictable pharmacokinetics and pharmacodynamics, limited potential for drug to drug interactions and can be given at fixed doses, without the need for routine coagulation monitoring [1]. They have been shown to have similar or improved efficacy and safety with VKAs across several thromboembolic disorders and act by specifically targeting a single coagulation factor, such as Factor Xa or thrombin [2]. Factor Xa is at the convergence point of the intrinsic and extrinsic coagulation pathways and it directly converts prothrombin to thrombin via the prothrombinase complex, leading to fibrin clot formation and platelet activation [3]. Factor Xa is, thus, a promising target for effective anticoagulation. Rivaroxaban is a direct Factor Xa inhibitor and has been approved for the prevention of venous thromboembolism in adults undergoing hip or knee replacement surgery, the prevention of stroke and systemic embolism in adults with non-valvular atrial fibrillation and for the treatment of deep vein thrombosis (DVT) and pulmonary embolism (PE), as well as the prevention of recurrent DVT and PE in adult patients [2]. The aim of this review is to provide an overview of the mechanism of action of rivaroxaban and outline its pharmacokinetic properties (absorption, distribution, metabolism, and excretion) in healthy subjects.

\section{Review}

\section{Mechanism of action}

In vitro kinetic analysis indicated that rivaroxaban is a selective, reversible, direct Factor Xa inhibitor that does not require cofactors for its anticoagulant effect [3-4]. It was found to be highly selective for human Factor Xa, with an inhibitory effect of 10,000 times higher than that for other serine proteases and an inhibition constant $\left(\mathrm{K}_{\mathrm{i}}\right)$ of $0.4 \mathrm{nM}$ [3]. Unlike indirect Factor Xa inhibitors, such as fondaparinux, rivaroxaban inhibits clot-bound Factor Xa (half-maximal inhibitory concentration ( $\left.\mathrm{IC}_{50}\right) 75 \mathrm{nM}$ ), as well as prothrombinase activity ( $\mathrm{IC}_{50} 2.1 \mathrm{nM}$ ), thereby prolonging clotting times. In human plasma, rivaroxaban has been shown to inhibit thrombin production and, thus, the amplification processes of coagulation, through the inhibition of endogenous Factor $\mathrm{Xa}\left(\mathrm{IC}_{50}\right.$ of $\left.21 \mathrm{nM}\right)[3,5]$. Therapeutically relevant concentrations of rivaroxaban (80-100 $\mathrm{nM}$ ) are sufficient to cause almost complete inhibition of thrombin generation [6]. In addition, rivaroxaban increases clot permeability and degradability, which may also contribute to its antithrombotic effect [7].

\section{Pharmacokinetics}

Absorption

A study by Kubitza et al. showed that after single-dose administration of rivaroxaban oral tablets (1.25$80 \mathrm{mg})$ in the fasted state, absorption was rapid and peak plasma concentration $\left(\mathrm{C}_{\max }\right)$ was reached after 2 hours [8]. Increases in $C_{\max }$ and area under the curve (AUC) were dose-proportional up until $10 \mathrm{mg}$ doses, 
but less than dose-proportional above $10 \mathrm{mg}$. This might be due limited solubility causing incomplete absorption of higher doses of rivaroxaban tablets, which would be consistent with the lower proportion of drug excreted unchanged in the urine at high doses $(60,80 \mathrm{mg})$ compared to the lowest dose $(1.25 \mathrm{mg})$ in this study (10\% compared to $40 \%$, respectively). However, a potential benefit of the decreased bioavailability at high doses might be the reduced risk of unintentional overdosing. Although this study was randomized, blinded, and placebo controlled, it only included 108 healthy white male subjects, aged 19 to 45 years with normal body mass index (BMI) and therefore may have been subject to selection bias, as about half of the patients on rivaroxaban are females, their median age is 74 years and their mean BMI is 28 [9]. Despite that, age, gender, ethnicity, and BMI were not shown to influence the absorption of rivaroxaban [10-12].

Another placebo-controlled and blinded dose escalation study looking at the effect of multiple-dose regimens (up to $30 \mathrm{mg}$ twice daily) in 61 healthy Caucasian male subjects aged 20 to 45 years, found that $\mathrm{C}_{\max }$ was reached 3 to 4 hours after the initial administration for all doses and all regimens and that at steady state [13]. Nevertheless, the AUC and $C_{\max }$ were dose-proportional for all doses, as opposed to the above study [8]. However, in this study rivaroxaban was taken in the fed state. The small sample size and possible selection bias limited the accuracy of the results. Another weakness could be the unequal randomization of patients to either drug or placebo ( $2: 1$ ratio), as it is possible that when outcome assessors are not agnostic about allocation (there is higher probability that a patient will fall under the intervention group), both positive and negative events might be altered [14].

The effect of food on the absorption and pharmacokinetics of rivaroxaban was investigated in a set of six independent, single-dose, cross-over studies in healthy male subjects [15]. Absorption was shown to be almost complete, with high oral bioavailability of greater than $80 \%$ in doses up to $10 \mathrm{mg}$, while the AUC and $\mathrm{C}_{\text {max }}$ were dose-proportional regardless of fasted or fed conditions. However, under fasting conditions, the pharmacokinetic parameters of $15 \mathrm{mg}$ and $20 \mathrm{mg}$ increased with dose but were less than doseproportional, with decreased bioavailability and absorption rate with increasing dose, consistent with Kubitza et al. [8]. For example, bioavailability dropped to $66 \%$ for the $20 \mathrm{mg}$ dose under fasting conditions. When rivaroxaban was taken with food however, dose proportionality and high bioavailability (more than 80\%) of these high doses were achieved. For instance, the mean AUC and $\mathrm{C}_{\max }$ of the $20 \mathrm{mg}$ dose increased by $39 \%$ and $76 \%$, respectively with food compared to fasted. The increased absorption in the fed state might relate to the lipophilicity and limited aqueous solubility of rivaroxaban. Finally, the absorption of rivaroxaban was unaffected by drugs altering gastric $\mathrm{pH}$, such as H2-receptor antagonists or antacids [16].

Distribution

In vitro protein binding in human plasma for rivaroxaban was found to be high (95\%) and reversible [17]. Experiments on isolated human plasma proteins revealed that serum albumin was the most significant plasma binding component (free fraction of rivaroxaban, $\mathrm{f}_{\mathrm{u}}$, was $19.6 \%$ ) and then $\mathrm{a}_{1}$-acid glycoprotein $\left(\mathrm{f}_{\mathrm{u}}=\right.$ 67.7\%). The discrepancy between the fuin human plasma (5.1\%) and in human serum albumin (19.6\%) could be explained by the presence of free fatty acids, whose binding might cause conformational changes in the albumin structure, therefore, increasing the protein binding of rivaroxaban. Moreover, a concentrationdependent increase in the fuof rivaroxaban was observed at concentrations above $10 \mathrm{mg} / \mathrm{L}$, possibly due to saturation of binding sites. However, the above in vitro results may not accurately predict what happens in vivo. The apparent volume of distribution $\left(\mathrm{V}_{\mathrm{SS}}\right)$ was estimated at $55 \mathrm{~L}$ from a population modeling analysis of rivaroxaban's pharmacokinetics, which used data from young, healthy subjects included in phase I trials, also indicating that it is mainly distributed to plasma, with a moderate affinity to peripheral tissues [18]. The accuracy of the model and its ability to provide mean population estimates is, however, debatable, given the small amount of data used. Rivaroxaban's moderate tissue affinity could be due to its moderate lipophilicity, as reflected in its partition coefficient value $(\log \mathrm{P}=1.5)$ [1]. This might explain the insignificant effect of body weight on the pharmacokinetics of rivaroxaban (and thus the fixed-dosing regimens regardless of body weight), as it is unlikely that total vascular bed size and blood volume will vary as much between subjects as body weight itself, which is mainly affected by the amount of body fat [11].

Metabolism

The metabolism of rivaroxaban was evaluated by Weinz et al. with the use of high-performance liquid chromatography and radioactivity detection by liquid scintillation counting [19]. The percentage of the initial oral dose that was found to undergo metabolic degradation was $57 \%$. Rivaroxaban was metabolized by both cytochrome P450 enzymes (CYP3A4/5, CYP2J2) and CYP-independent mechanisms. CYP3A4 accounted for $18 \%$ and CYP2J2 for $14 \%$ of total rivaroxaban elimination [19-20]. In addition to this oxidative biotransformation, non-CYP-mediated hydrolysis of the amide bonds accounted for $14 \%$ of total rivaroxaban elimination. No major or active metabolites were detected in plasma and unchanged rivaroxaban was the most important compound in human plasma (89\% of total AUC), reflecting a minimal presence of metabolites. In fact, the plasma concentration-time curve for unchanged rivaroxaban was very similar to that for total radioactivity, meaning that the pharmacological effects of rivaroxaban were largely due to the unchanged drug. The predominant metabolite M-1 accounted for only $3 \%$ of total plasma radioactivity. In this study, however, only four young male subjects of normal BMI were used, limiting the 
credibility and perhaps the generalisability of the findings. In vitro structural studies in liver microsomes and hepatocytes of humans showed that M-1 was produced through oxidative degradation of the morpholinone moiety of rivaroxaban (forming M2), followed by oxidative cleavage of the ring [20]. Oxidative degradation of the morpholinone ring also produced the minor metabolites M-5 and M-6. Metabolite M-4 was produced by hydrolysis of the amide bond of rivaroxaban, with subsequent conjugation of M-13 with glycine. Hydrolytic cleavage of the lactam amide bond in the morpholinone moiety resulted in M-7. NADPHindependent hydrolysis of the amide bond of rivaroxaban formed M-15, which underwent NADPHindependent oxidation to form M-16 via monoamine oxidases in human liver microsomes. M-16 was then either reduced to the alcohol derivative M-17 or further oxidized to the carboxylic acid derivative M-18. Clearly, in vitro studies are inherently flawed by nature, as they may not accurately describe the metabolism in vivo. For example, this study excluded the possibility that extra-hepatic enzymes might play a role in the metabolic degradation of rivaroxaban, since only hepatocytes or liver microsomes were used.

In summary, the metabolism of rivaroxaban was shown to occur via two major pathways: oxidative degradation of the morpholinone moiety (via CYP450) and hydrolysis of the different amide bonds (CYPindependent). Given then role of CYP450 in rivaroxaban's metabolism, it would be reasonable to assume that inhibitors or inducers of these enzymes would affect the drug's pharmacokinetics. Indeed, Mueck et al. demonstrated that concomitant administration of rivaroxaban with strong inhibitors of both CYP3A4 and Pglycoprotein/breast cancer resistance protein (P-gp/BCRP) (such as ketoconazole and ritonavir), significantly increased rivaroxaban exposure [21]. By inhibiting CYP3A4, the metabolism of rivaroxaban will be reduced and thus the plasma concentration of the active, unchanged drug will be increased. However, strong inhibitors of either CYP3A4 or P-gp, or moderate inhibitors of both of these pathways, produced less marked effects. Finally, due to the highly polymorphic nature of CYP450s, a degree of inter-individual variability is expected in the metabolism of rivaroxaban, but more studies are necessary to evaluate the magnitude of the effect of CYP450 single nucleotide polymorphisms on rivaroxaban's pharmacokinetics [22].

Excretion

Weinz et al. showed that excretion occurred predominantly via the renal route (66\%) and to a lesser extent via the faecal/biliary routes (28\%), by measuring rivaroxaban associated radioactivity in humans [19]. The percentage of the initial dose excreted as unchanged drug was $43 \%$, of which $36 \%$ was excreted via urine and $7 \%$ via the feces. Of the $36 \%$ of the rivaroxaban dose eliminated in urine, active renal secretion accounted for approximately $30 \%$ and glomerular filtration for the rest [18-19]. According to in vitro and in vivo drug studies, the transporters involved in active renal secretion of rivaroxaban are P-gp and BCRP. Inhibiting Pgp/BCRP would therefore result in reduced renal clearance and enhanced drug exposure, as discussed above $[21,23]$. In addition, $30 \%$ and $21 \%$ of the initial dose was eliminated as metabolites in the urine and feces, respectively. Mean terminal half-life of about six to nine hours and apparent oral clearance of about 10 $\mathrm{L} /$ hour were found in young healthy subjects in doses up to $20 \mathrm{mg}[8,13]$. In a study of healthy subjects of 75 years old and over however, half-life was found to be 11 to 12 hours, whereas total and renal clearance inversely correlated with age, but positively with creatinine clearance, which is age-dependent and a marker of renal function [10]. The reduced rivaroxaban clearance in the elderly subjects, as a result of the expected and well-documented reduction in renal function with increasing age, led to a statistically significant impact on the exposure of rivaroxaban [24]. Nevertheless, dose-adjustments based on age alone would not be necessary, as age was insufficient to explain the increased drug exposure on its own, but may be required based on renal function. A limitation of this study was once again the small sample size ( $n=34)$, but an important strength in contrast to the other studies, was the equal representation of both genders, as well as the greater range of BMIs. The above is in agreement with studies in patients with renal impairment, which have demonstrated that renal impairment results in decreased renal clearance of a single rivaroxaban $10 \mathrm{mg}$ oral dose and increased overall exposure to the drug [25].

\section{Conclusions}

In this review, the mechanism of action and the pharmacokinetic properties of rivaroxaban in healthy subjects were outlined. Rivaroxaban is a direct, reversible and highly selective factor Xa inhibitor that inhibits thrombin generation at therapeutically relevant concentrations. Overall, in healthy subjects, rivaroxaban was shown to exhibit predictable and dose-proportional pharmacokinetics in the fed state without dose accumulation beyond a steady state. Demographic variations had little influence on rivaroxaban's pharmacokinetics, except for age, but its effect was found to be insignificant after adjusting for renal function. Furthermore, co-administration of rivaroxaban with strong inhibitors of both CYP3A4 and P-gp was identified to cause a clinically relevant increase in drug exposure, which was expected given their role in the drug's metabolism and excretion. Finally, it is important to note that, since this review aimed to examine the pharmacokinetics of rivaroxaban in healthy volunteers, the majority of the studies evaluated were phase I and had significant limitations, as discussed. To summarise, the high oral bioavailability, the low-to-moderate potential for peripheral tissue penetration, combined with the absence of major or active metabolites in human plasma and its relatively short half-life, highlight rivaroxaban's favorable pharmacokinetics and justify its central role in current anticoagulation management across several thromboembolic conditions. 


\section{Additional Information \\ Disclosures}

Conflicts of interest: In compliance with the ICMJE uniform disclosure form, all authors declare the following: Payment/services info: All authors have declared that no financial support was received from any organization for the submitted work. Financial relationships: All authors have declared that they have no financial relationships at present or within the previous three years with any organizations that might have an interest in the submitted work. Other relationships: All authors have declared that there are no other relationships or activities that could appear to have influenced the submitted work.

\section{References}

1. Mueck W, Stampfuss J, Kubitza D, Becka M: Clinical pharmacokinetic and pharmacodynamic profile of rivaroxaban. Clin Pharmacokinet. 2014 , 53:1-16. 10.1007/s40262-013-0100-7

2. Ageno W, Gallus AS, Wittkowsky A, Crowther M, Hylek EM, Palareti G: Oral anticoagulant therapy: antithrombotic therapy and prevention of thrombosis, 9th ed: American college of chest physicians evidence-based clinical practice guidelines. Chest. 2012, 141:e44S-e88S. 10.1378/chest.11-2292

3. Perzborn E, Strassburger J, Wilmen A, Pohlmann J, Roehrig S, Schlemmer K-H, Straub A: In vitro and in vivo studies of the novel antithrombotic agent BAY 59-7939-an oral, direct Factor Xa inhibitor. J Thromb Haemost. 2005, 3:514-21. 10.1111/j.1538-7836.2005.01166.x

4. Samama MM: The mechanism of action of rivaroxaban-an oral, direct Factor Xa inhibitor-compared with other anticoagulants. Thromb Res. 2011, 127:497-504. 10.1016/j.thromres.2010.09.008

5. Siddiqui F, Hoppensteadt D, Jeske W, Iqbal O, Tafur A, Fareed J: Factor xa inhibitory profile of apixaban, betrixaban, edoxaban, and rivaroxaban does not fully reflect their biologic spectrum. Clin Appl Thromb Hemost. 2019, 25:1-11. 10.1177/1076029619847524

6. Gerotziafas GT, Elalamy I, Depasse F, Perzborn E, Samama MM: In vitro inhibition of thrombin generation, after tissue factor pathway activation, by the oral, direct factor Xa inhibitor rivaroxaban. J Thromb Haemost. 2007, 5:886-8. 10.1111/j.1538-7836.2007.02429.x

7. Varin R, Mirshahi S, Mirshahi P, et al.: Effect of rivaroxaban, an oral direct factor xa inhibitor, on whole blood clot permeation and thrombolysis: critical role of red blood cells. Blood. 2009, 114:1064.

8. Kubitza D, Becka M, Voith B, Zuehlsdorf M, Wensing G: Safety, pharmacodynamics, and pharmacokinetics of single doses of BAY 59-7939, an oral, direct factor Xa inhibitor. Clin Pharmacol Ther. 2005, 78:412-21. 10.1016/j.clpt.2005.06.011

9. Beyer-Westendorf J, Förster K, Pannach S, et al.: Rates, management, and outcome of rivaroxaban bleeding in daily care: results from the Dresden NOAC registry. Blood. 2014, 124:955-62. 10.1182/blood-2014-03563577

10. Kubitza D, Becka M, Roth A, Mueck W: The influence of age and gender on the pharmacokinetics and pharmacodynamics of rivaroxaban-an oral, direct factor Xa inhibitor. J Clin Pharmacol. 2013, 53:249-55. 10.1002/jcph.5

11. Kubitza D, Becka M, Zuehlsdorf M, Mueck W: Body weight has limited influence on the safety, tolerability, pharmacokinetics, or pharmacodynamics of rivaroxaban (bay 59-7939) in healthy subjects. I Clin Pharmacol. 2007, 47:218-26.

12. Zhao X, Sun P, Zhou Y, et al.: Safety, pharmacokinetics and pharmacodynamics of single/multiple doses of the oral, direct Factor Xa inhibitor rivaroxaban in healthy Chinese subjects. Br J Clin Pharmacol. 2009, 68:77-88. 10.1111/j.1365-2125.2009.03390.x

13. Kubitza D, Becka M, Wensing G, Voith B, Zuehlsdorf M: Safety, pharmacodynamics, and pharmacokinetics of BAY 59-7939-an oral, direct Factor Xa inhibitor-after multiple dosing in healthy male subjects. Eur J Clin Pharmacol. 2005, 61:873-80. 10.1007/s00228-005-0043-5

14. Hey SP, Kimmelman J: The questionable use of unequal allocation in confirmatory trials . Neurology. 2014, 82:77-9. 10.1212/01.wnl.0000438226.10353.1c

15. Stampfuss J, Kubitza D, Becka M, Mueck W: The effect of food on the absorption and pharmacokinetics of rivaroxaban. Int J Clin Pharmacol Ther. 2013, 51:549-61. 10.5414/CP201812

16. Kubitza D, Becka M, Zuehlsdorf M, Mueck W: Effect of food, an antacid, and the $\mathrm{H} 2$ antagonist ranitidine on the absorption of BAY 59-7939 (rivaroxaban), an oral, direct factor Xa inhibitor, in healthy subjects. J Clin Pharmacol. 2006, 46:549-58. 10.1177/0091270006286904

17. Weinz C, Buetehorn U, Daehler H-P, et al.: Pharmacokinetics of BAY 59-7939-an oral, direct Factor Xa inhibitor-in rats and dogs. Xenobiotica. 2005, 35:891-910.

18. Mueck W, Becka M, Kubitza D, Voith B, Zuehlsdorf M: Population model of the pharmacokinetics and pharmacodynamics of rivaroxaban-an oral, direct factor xa inhibitor-in healthy subjects. Int J Clin Pharmacol Ther. 2007, 45:335-44. 10.5414/cpp45335

19. Weinz C, Schwarz T, Kubitza D, Mueck W, Lang D: Metabolism and excretion of rivaroxaban, an oral, direct factor Xa inhibitor, in rats, dogs, and humans. Drug Metab Dispos. 2009, 37:1056-64. 10.1124/dmd.108.025569

20. Lang D, Freudenberger C, Weinz C: In vitro metabolism of rivaroxaban, an oral, direct factor Xa inhibitor, in liver microsomes and hepatocytes of rats, dogs, and humans. Drug Metab Dispos. 2009, 37:1046-55. 10.1124/dmd.108.025551

21. Mueck W, Kubitza D, Becka M: Co-administration of rivaroxaban with drugs that share its elimination pathways: pharmacokinetic effects in healthy subjects. Br J Clin Pharmacol. 2013, 76:455-66. 10.1111/bcp.12075

22. Preissner SC, Hoffmann MF, Preissner R, Dunkel M, Gewiess A, Preissner S: Polymorphic cytochrome P450 enzymes (CYPs) and their role in personalized therapy. PLoS One. 2013, 8:e82562. 10.1371/journal.pone.0082562

23. Gnoth MJ, Buetehorn U, Muenster U, Schwarz T, Sandmann S: In vitro and in vivo p-glycoprotein transport 


\section{Cureus}

characteristics of rivaroxaban. J Pharmacol Exp Ther. 2011, 338:372-80. 10.1124/jpet.111.180240

24. Denic A, Glassock RJ, Rule AD: Structural and functional changes with the aging kidney . Adv Chronic Kidney Dis. 2016, 23:19-28. 10.1053/j.ackd.2015.08.004

25. Kubitza D, Becka M, Mueck W, et al.: Effects of renal impairment on the pharmacokinetics,

pharmacodynamics and safety of rivaroxaban, an oral, direct Factor Xa inhibitor. Br J Clin Pharmacol. 2010, 70:703-12. 10.1111/j.1365-2125.2010.03753.x 